\title{
Cell Image Processing Methods for Automatic Cell Pattern Recognition and Morphological Analysis of Mesenchymal Stem Cells - An Algorithm for Cell Classification and Adaptive Brightness Correction -
}

\author{
Kitaek Lim ${ }^{1,3}$, Soo Hyun Park', Jangho Kim¹, Hoon Seonwoo ${ }^{1}$, Pill-Hoon Choung ${ }^{3,4}$, Jong Hoon Chung ${ }^{1,2 *}$ \\ ${ }^{1}$ Department of Biosystems and Biomaterials Science and Engineering, Seoul National University, Seoul, Korea \\ ${ }^{2}$ Research Institute for Agriculture and Life Sciences, Seoul National University, Seoul, Korea \\ ${ }^{3}$ Department of Oral and Maxillofacial Surgery and Dental Research Institute, School of Dentistry, \\ Seoul National University, Seoul, Korea \\ ${ }^{4}$ Tooth Bioengineering National Research Laboratory of Post BK21, School of Dentistry, \\ Seoul National University, Seoul, Korea
}

Received: February $18^{\text {th }}, 2013$; Revised: February $28^{\text {th }}, 2013$; Accepted: February $28^{\text {th }}, 2013$

\section{Abstract}

Purpose: The present study aimed at image processing methods for automatic cell pattern recognition and morphological analysis for tissue engineering applications. The primary aim was to ascertain the novel algorithm of adaptive brightness correction from microscopic images for use as a potential image analysis. Methods: General microscopic image of cells has a minor problem which the central area is brighter than edge-area because of the light source. This may affect serious problems to threshold process for cell-number counting or cell pattern recognition. In order to compensate the problem, we processed to find the central point of brightness and give less weight-value as the distance to centroid. Results: The results presented that microscopic images through the brightness correction were performed clearer than those without brightness compensation. And the classification of mixed cells was performed as well, which is expected to be completed with pattern recognition later. Beside each detection ratio of hBMSCs and HeLa cells was 95\% and 92\%, respectively. Conclusions: Using this novel algorithm of adaptive brightness correction could control the easier approach to cell pattern recognition and counting cell numbers.

Keywords: Image processing, Cell pattern recognition, Morphological analysis, Mesenchymal stem cells, Tissue engineering applications

\section{Introduction}

Stem cells have attracted tremendous interest in recent times due to their promise in providing innovative treatments for debilitating some disease. This is due to their potential ability to regenerate and repair damaged tissues. In particular, human bone marrow-derived mesenchymal stem cells (hBMSCs) are one type of adult stem cells. Despite the attractive properties of hBMSCs, there is

\footnotetext{
*Corresponding author: Jong Hoon Chung

Tel: +82-2-880-4601; Fax: +82-2-880-4601

E-mail: jchung@snu.ac.kr
}

presently no quick and easy way to find out the various cells. Until now, a lot of time is needed in order to search for abnormal cells, and it is easy to be subject to various time-consuming assays. Hence there is a great need for innovative new ways to assess the cell classification, as well as the quality of cell cultures for potential clinical application. The research presented in the paper investigates the use of digital image processing and pattern recognition techniques to provide a quick and simple method for the assessment of hBMSCs. Above all, the aim of this work is to ascertain whether it is possible, through the use of cell image processing and pattern recognition techniques. 
Several researchers have been developing automated methods for segmenting and counting cells in microscopic images (Spencer et al., 1996; Garrido etl al., 2000; Shiotani et al., 1994). Some approaches are based on machine learning. Long et al. (2005) and Zheng et al. (2004) proposed methods based on neural network and Markiewicz et al. (2006) proposed a method to cell recognition and count using Support Vector Machine. In this kind of approach, the major task is to create the learning set, which is usually done manually by an independent expert for cell type. With the discovery of the potential of stem cells, many researches have been dealing with this kind of cell. Althoff et al. (2005) and Tang et al. (2005) proposed a method for segmentation and tracking of neural stem cells. Both approaches are based on classical segmentation methods and use the information about the cells' previous position to decide which blobs correspond to real cells. Also working with neural stem cells, Korzynska (2007) presented a method for automatic counting of neural stem cells growing in cultures which is performed in two steps: 1) segmentation step: the image is separated in several regions and; 2)counting step: each homogeneous region is counted separately. Kachouie et al. (2007) proposed a deconvolution method in the form of an optimized ellipse fitting algorithm to locate individuals hematopoietic stem cells. The methods proposed in (Kachouie et al., 2006; Kachouie et al., 2005) uses the cell morphologic information (e. g. cell size, boundary brightness, interior brightness and boundary uniformity or symmetry) to locate and track hematopoietic stem cells. Importantly the works cited above handled with only one type of stem cell in their images.

Morphological cell analysis has been integrated in new methods for biomedical applications, such as automatic segmentation and analysis of histological tumor sections (Reyes-Aldasoro et al., 2011; Cheng et al., 2010; Schildkraut et al., 2010), boundary detection of cervical cell nuclei considering overlapping and clustering (Plissiti et al., 2011), the granules segmentation and spatial distribution analysis (Diaz et al., 2010), and morphological characteristics analysis of specific biomedical cells (Brun et al., 2011; Amini et al., 2010; Xiong et al., 2010).

Especially, morphological feature quantification for grading cancerous or precancerous cells is widely researched in literatures, such as nuclei segmentation based on marker-controlled watershed transform and snake model for hepatocelluar carcinoma feature extraction and classification, which is important to prognosis and treatment planning (Huang et al., 2010), nuclei feature quantification for cancer cell cycle analysis (Li et al., 2010), and using feature extraction include image morphological analysis, wavelet analysis and texture analysis for automated classification of renal cell. (Chaudry et al., 2009) Computerized/automated early cancer or abnormalities detection provides a basis for reducing deaths and morbidity, especially for cervical cancer, which is reported to be the most preventable disease through early detection, provision of prompt advice and opportunities for follow-up treatments (Chang et al., 1996) .

The scope of this paper is restricted to morphological cell analysis by image processing in the field of tissue engineering research. As such, the goal of this study was to investigate the image processing methods for automatic cell pattern recognition and morphological analysis for tissue engineering applications. The primary aim is to ascertain the novel algorithm of adaptive brightness correction from microscopic images for use as a potential image analysis. In addition, the ability to automatic cell pattern recognition and morphological analysis was investigated and established an adaptive calibration model in an attempt to indicate a possibility of image processing techniques.

\section{Materials and Methods}

\section{Culture of hBMSCs and HeLa cells}

hBMSCs were obtained from the Intellectual Biointerface Engineering Center, Dental Research Institute, College of Dentistry, Seoul National University. Also, HeLa cells, cancer cell line, were grown on Petri dishes. Cells were cultured in alpha-minimum essential medium ( $\alpha$-MEM, LM 008-01, Welgene Inc., South Korea) containing $10 \%$ fetal bovine serum (FBS, Welgene Inc., South Korea), $10 \mathrm{mM}$ ascorbic acid (L-ascorbic acid), antibiotics, and sodium bicarbonate at $37^{\circ} \mathrm{C}$ in a humidified atmosphere of $5 \% \mathrm{CO}_{2}$ (Steri-Cycle 370 Incubator, Thermo Fisher Scientific, USA). Cell culture was initiated with a cell seeding density of $3.0 \times 10^{4}$ viable cells per well. Representative cell images of the two types were prepared in this study (Fig. 1). In the cause of brightness correction, the novel normalization method for microscopic image was developed. Continuously, threshold process was applied to the next step such as cell number counting and specific pattern recognition of various cells. 


\section{Brightness compensation of microscopic images}

General microscopic image of cells has a minor problem that the central area is brighter than edge-area because of the light source by inverted microscope. This may affect serious problems to threshold process for cellnumber counting or cell pattern recognition. In order to compensate effectively the problem, we processed to find the central point of brightness and give less weight-value as the distance to centroid. Using this new algorithm of adaptive brightness correction could control the easier approach to cell pattern recognition and counting cell numbers. Figure 2 showed the procedure of cell image processing method for adaptive brightness correction from microscopic images and related equations.

Brightness compensation algorithm was completed with adding the weight value which was set in proportion to the distance from the brightness center. Gap in Eq. 2 is assigned to the difference between the average pixel values of manually selected the brightest and darkest points and it is divided by half of diagonal length (HD) in Eq. 2 in order to avoid becoming too large for weight value. In detail, the brightness correction algorithm has thresholding for background elimination, erosion for
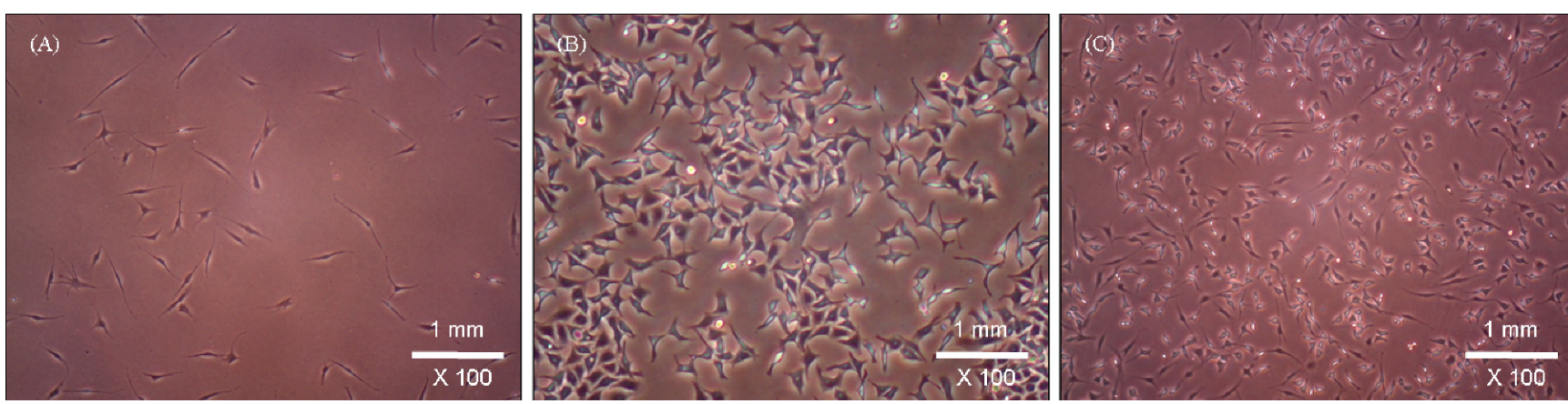

Figure 1. Representative cell images for automatic cell pattern recognition and morphological analysis between hBMSCs and HeLa cells.
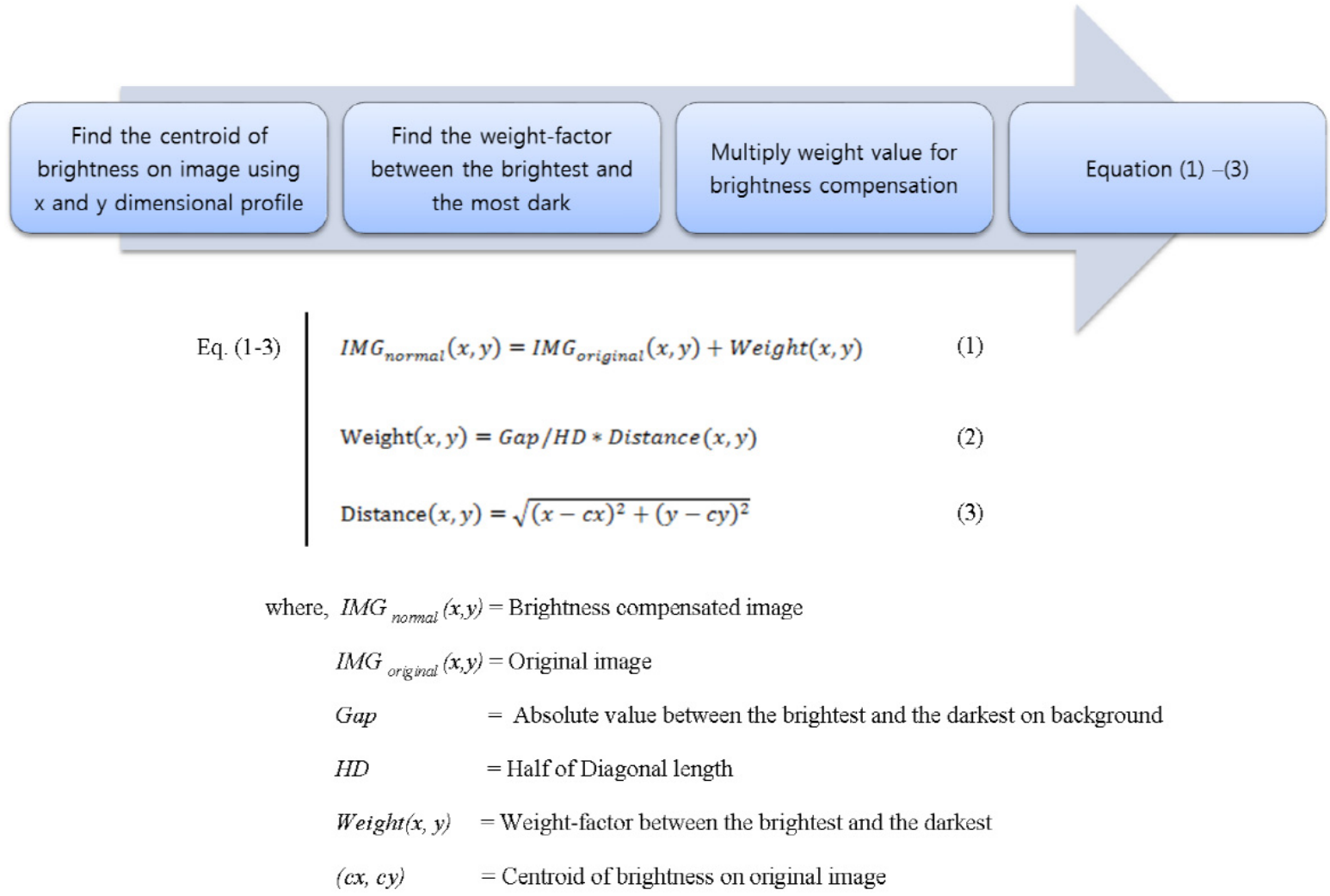

Figure 2. Brightness normalization procedures for compensation of pre-processing from microscopic images and related equations. 
noise removal and labeling for object classification. In order to compensate the difference of brightness between center and edge, weight value was added to gray level at each pixel. The maximum weight was set less than the absolute value between the brightest and the darkest to keep the corrected gray level less than 255. Adaptive brightness correction algorithm from microscopic images was developed in this study, and then calculated as following Eq. (1)-(3).

Figure 3 indicates methods performed in this study for cell classification. The fundamental principle of morphological cell analysis is dependent on cell patterns. To study cell characteristics, the procedure to detect abnormalities such as HeLa cell and determine the hBMSCs under a microscope was used in Fig. 3. For carrying out the algorithm, the function region props in MATLAB (R2011b, Mathworks Inc., Natick, MA, USA) was used to measure properties of a selected region of an image as ellipse shape. Before applying the function region props, the actual image needs to be converted into a binary image and labeled image for numbering each cell. Function called rgb2gray and bwlabel, were used to separate background and cells area in images. This labeled region underwent image erosion and dilution procedures to remove noises and be seen clear.

The developed algorithm has the following features

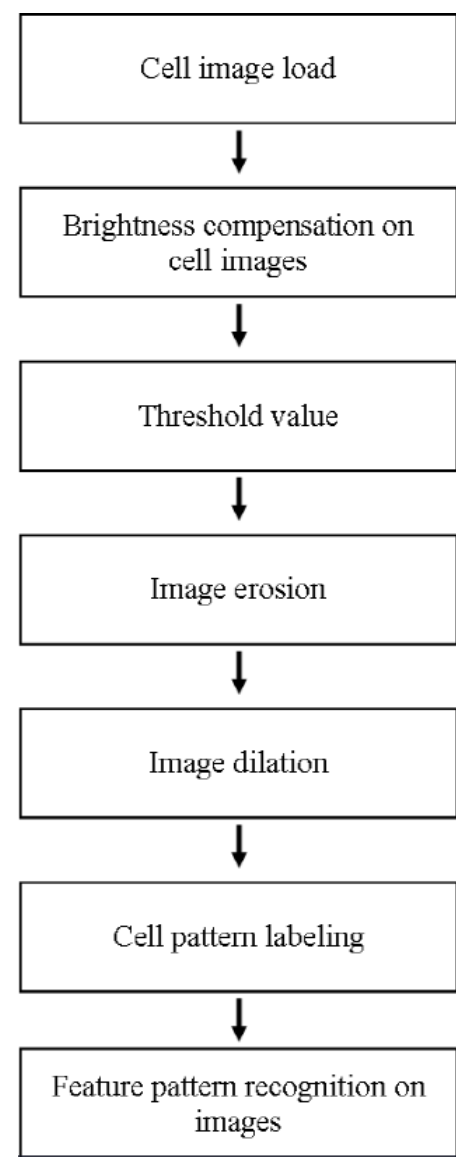

Figure 3. The procedure of image processing method for labeling the featured pattern recognition from microscopic images.

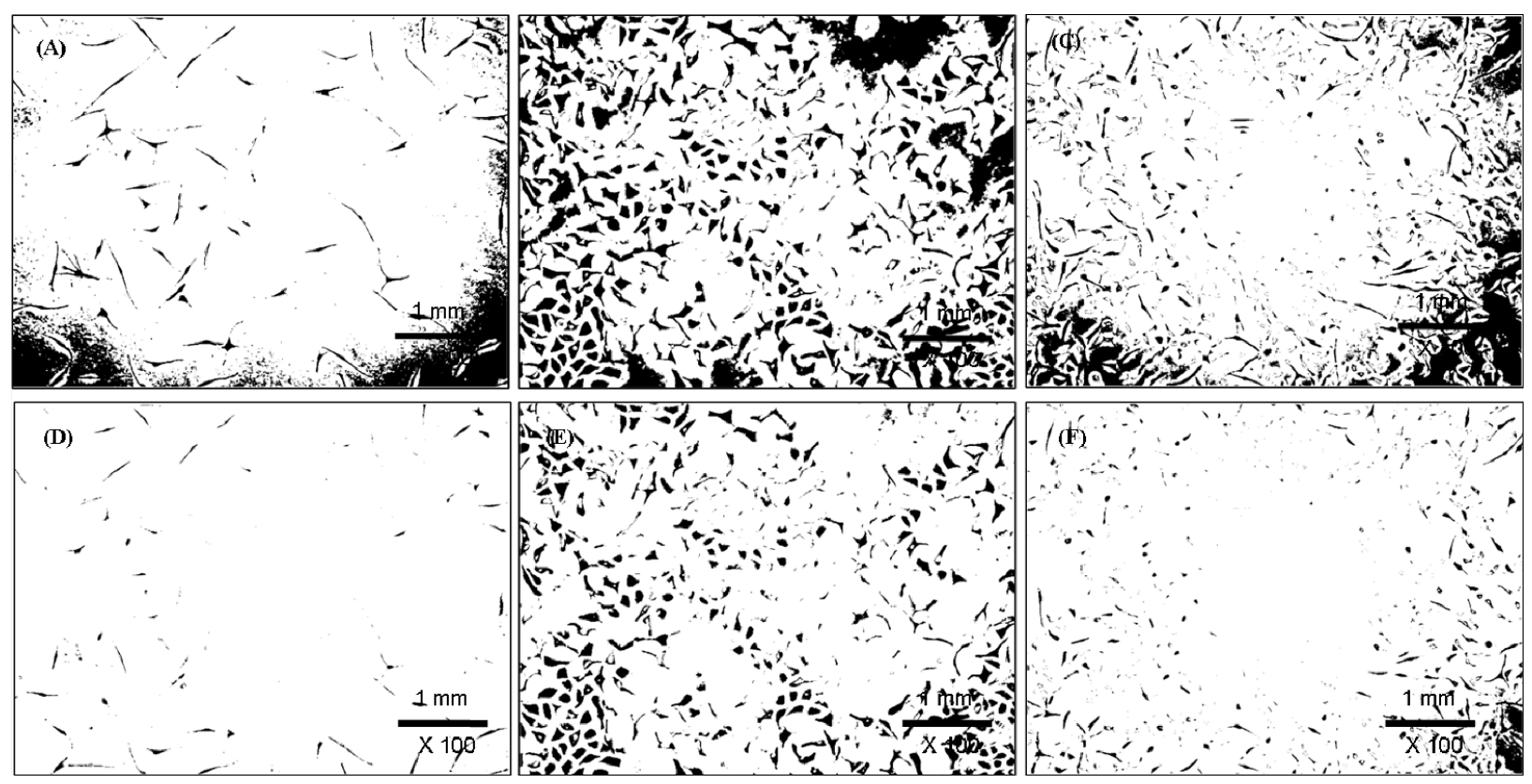

Figure 4. Possible problems after binary images process in conventional method; the edge area of microscopic images became to black because it was darker than the center in the original image of MSCs cells (A), HeLa cells (B) and co-cultured cells (C). The same original images of hBMSCs $(D)$, HeLa cells $(E)$ and co-cultured cells $(F)$ were treated with a different threshold value, but the trial to remove of edge background led the cell missing in the central area. 
compared to the conventional method. In the conventional method, there is no specific process to distinguish the most dark and bright area of the background of acquired micrographs. Problems occur in the process of binary image, which are reorganizing all outlying area as background or reorganizing the brighter center area as a large cell. Therefore the algorithm focused on settling those troubles as shown in Fig. 4 (A-F). It works by selecting the most dark and bright pixel of the microscope image manually, and then it makes the brightness level of the two selected points to the similar level. The binary image of the brightness-compensated microscopic images showed remarkable improvement.

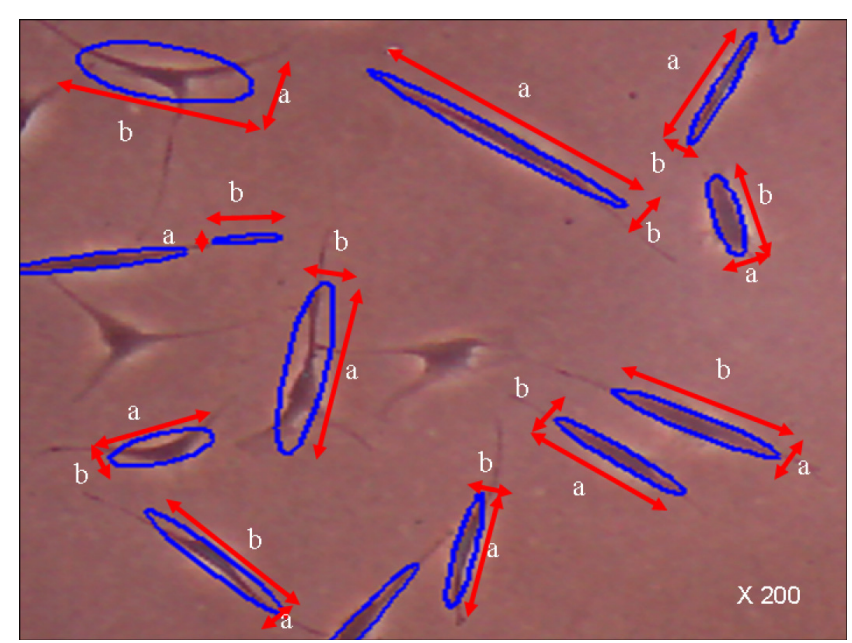

Figure 5. Representative feature pattern recognition analysis for cell image processing.

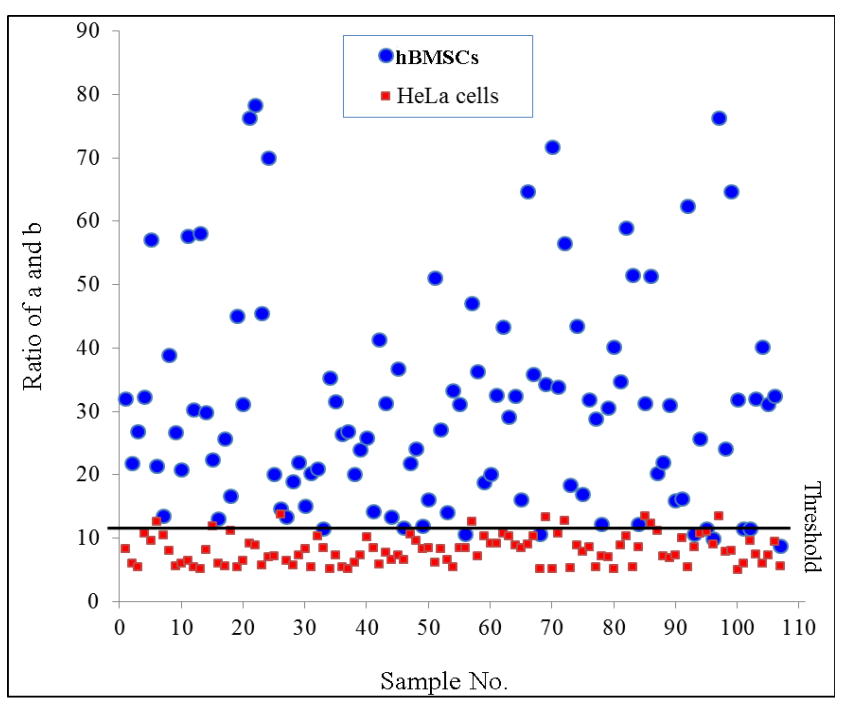

Figure 6. The determination of threshold value to separate normal cell and cancer cell using the ratio. The error was the lowest when the threshold was 12 shown as dot line.

\section{Condition of feature pattern recognition}

Figure 5 shows how to calculate the ratio of each cell. The lengths of major and minor axis of ellipse covering cell were calculated first, and the greater ratio values among $\mathrm{a} / \mathrm{b}$ and $\mathrm{b} / \mathrm{a}$ were considered as the ratio. As shown in cell morphologies, hBMSCs indicated cell shapes were longer than those of HeLa cells. In this study, we decided threshold value for distinguishing hBMSCs and HeLa cells, respectively. The hBMSCs is marked and indicated as blue pattern when the ratio of major and minor axes are greater than 12 otherwise HeLa cell is marked and indicated as red pattern (Fig. 6).

\section{Results and Discussion}

\section{Feature pattern recognition of hBMSCs}

Figure 7 showed the resultant images by each processing step of hBMSCs: representative cell image (A); image after brightness compensation (B); image after threshold treatment $(\mathrm{C})$; image of cell feature pattern recognition (blue, D). Acquired microscopic hBMSCs cell image (Fig. 7-A) went through processing steps to visualize individual cell. The final resultant image on Fig. 7-(D) shows some marked cells which were classified as hBMSCs correctly and some missed cells which were not marked on image. It is determined that appeared some brighter cells and multi-directional growing cells caused the error detection. Brighter cells were removed when binary image process because of gray values close to background brightness and the ratio of multi-directional growing cells were near 1 which should be classified ad hBMSCs cells.

\section{Feature pattern recognition of HeLa cells}

Figure 8 indicated resultant images by each processing step of HeLa cells: representative cell image (A); image after brightness compensation (B); image after threshold treatment (C); image of cell feature pattern recognition (red mark, D). The most of HeLa cells are attached and formed clusters shown in Fig. 8-A. This caused a cluster to be recognized as big single cell and it led the ratio of the cell to be unusual. Furthermore, the brightness of background becomes darker relatively in the case of cells gathering. It interrupts the function of brightness compensation process. That is why image shown on Fig. 8-C represented the uniform brightness distribution on background overall. 

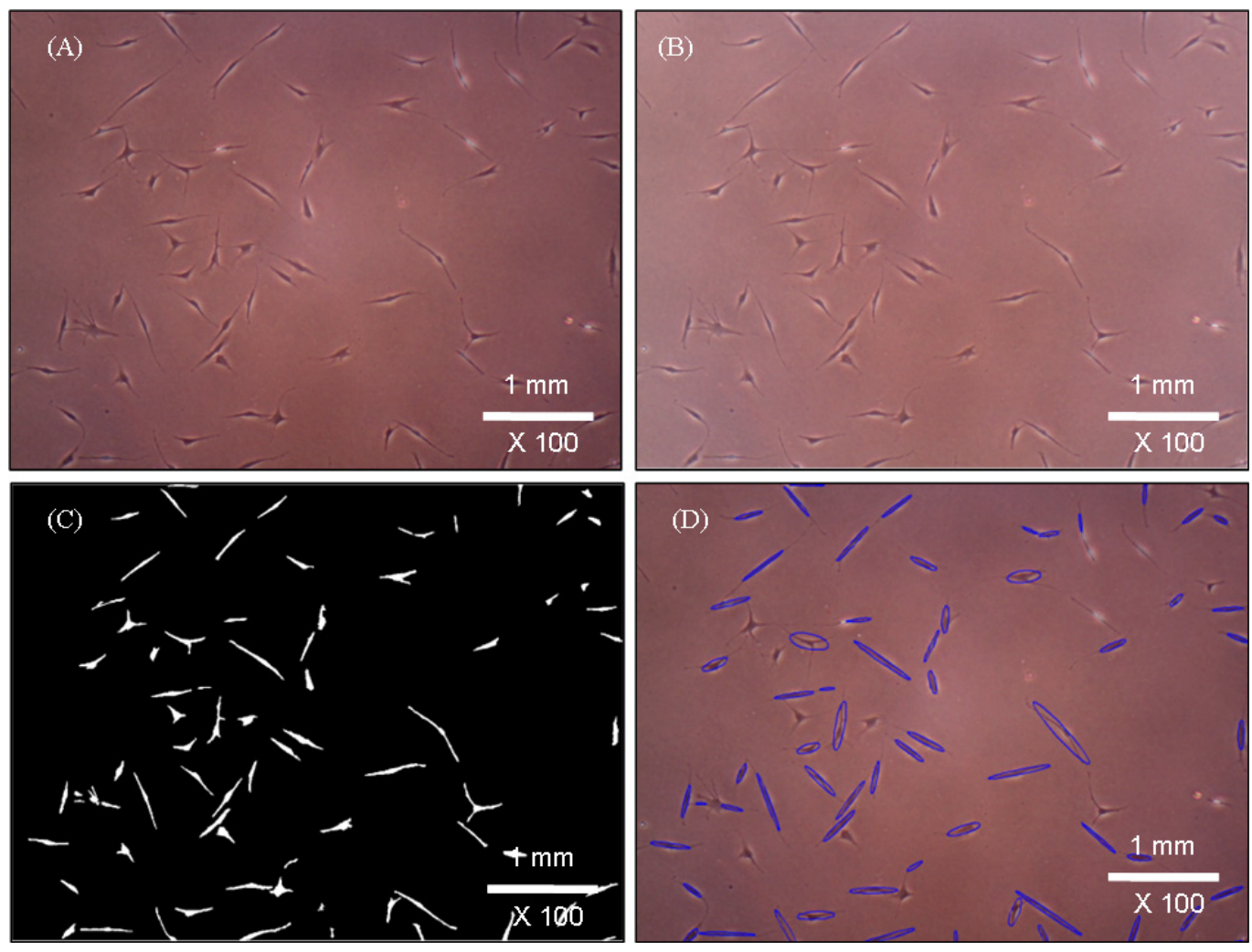

Figure 7. Resultant images by each processing step of hBMSCs: representative cell image (A); image after brightness compensation (B); image after threshold treatment $(C)$; image of cell feature pattern recognition (blue mark, $D$ ).
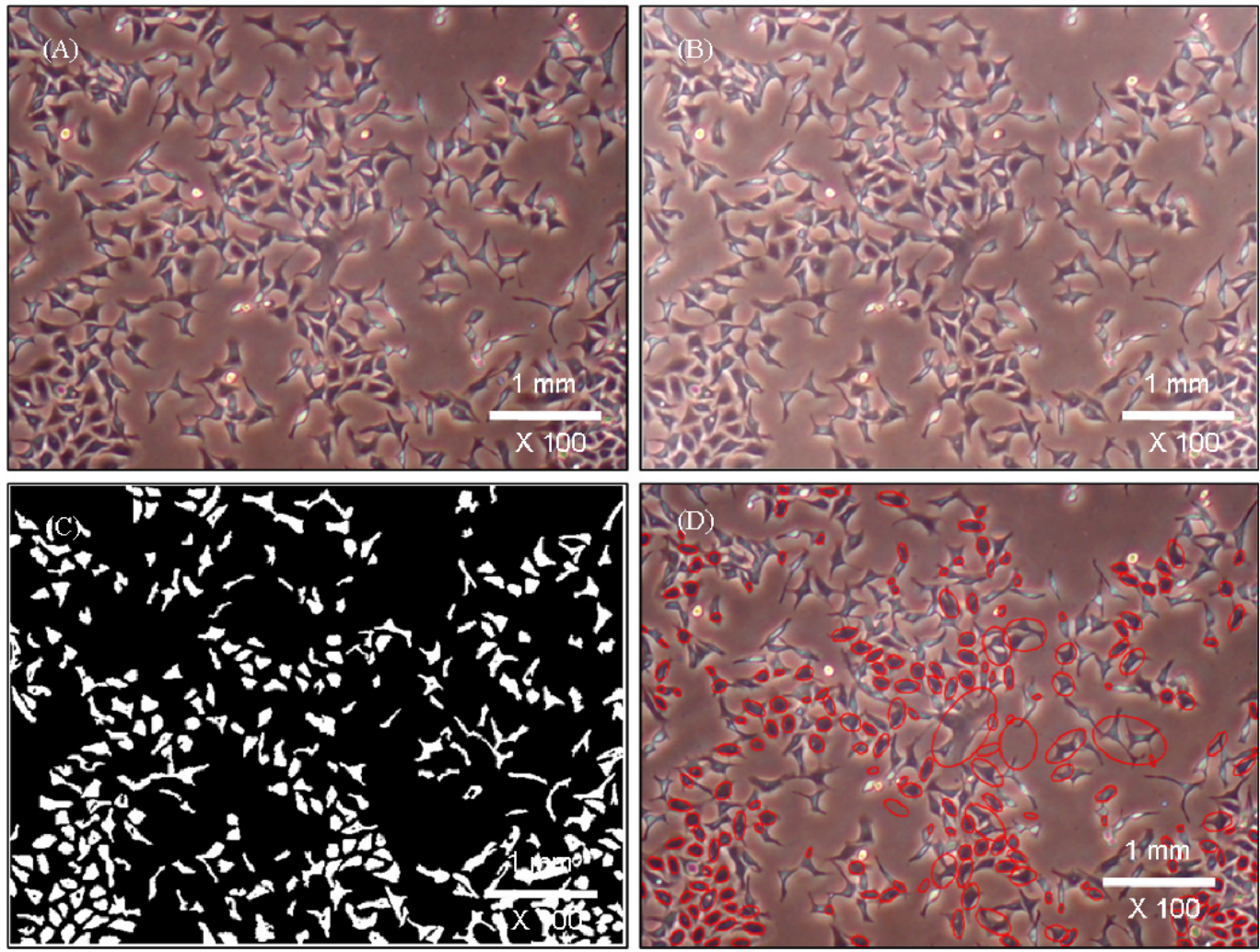

Figure 8. Resultant images by each processing step of HeLa cells: representative cell image (A); image after brightness compensation $(B)$; image after threshold treatment $(C)$; image of cell feature pattern recognition (red mark, D). 

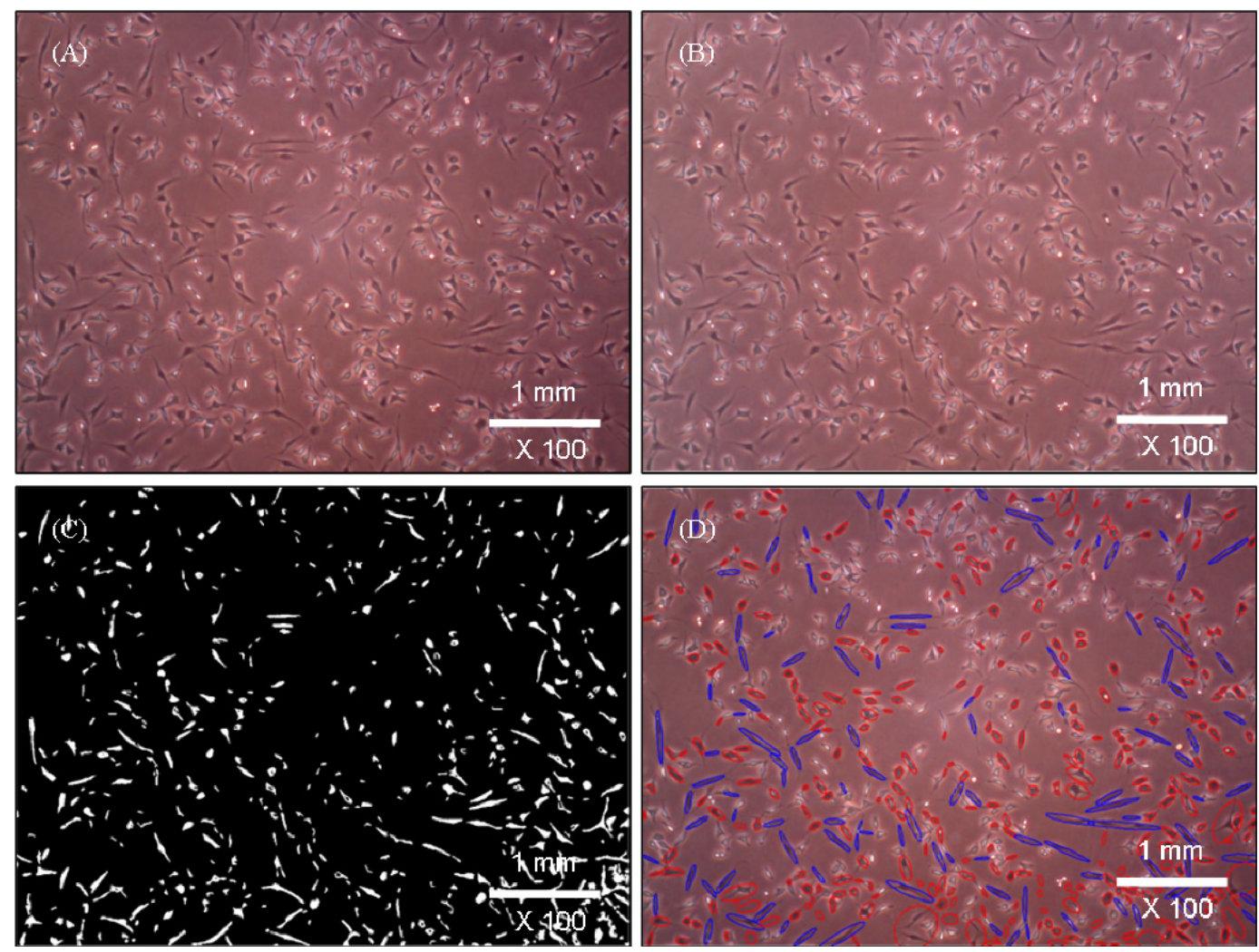

Figure 9. Resultant images by each processing step via co-culture of MSCs and HeLa cells: representative cell image (A); image after brightness compensation (B); image after threshold treatment $(C)$; image of cell feature pattern recognition (blue mark: hBMSCs; red mark: HeLa cells, D).

\section{Cell classification with hBMSCs and HeLa cells}

Figure 9 showed resultant images by each processing step via co-culture of hBMSCs and HeLa cells: representative cell image (A); image after brightness compensation (B); image after threshold treatment (C); image of cell feature pattern recognition (blue mark: hBMSCs; red mark: HeLa cells, D). Marking on image was completed with drawing ellipse bounding after thresholding and labeling process. This figure illustrates the two axes of the ellipse which are shown in Fig. 5 as a and b. The ratio of axes was determined as the length of major axis divided by that of minor axis. The determination of threshold value to separate hBMSCs and HeLa cells using the ratio was shown in Fig. 6. One hundred and ten standard cells of hBMSCs and HeLa cells respectively were investigated. The axes of ellipse were used to calculate the ratio. Based on the ratios, the optimal threshold value was determined to 12 which was shown that the error was shown as black line in Fig. 6. From the threshold value, we could successfully classify cell feature pattern recognition hBMSCs and HeLa cells. Beside each detection ratio of hBMSCs and HeLa cells was 95\% and 92\%, respectively.
The miss-detected cells are classified to 3 cases. The first is the case of cell recognition failure at which cell form disappear in the process of binary and erosion step because of small size or higher gray level relatively. The second case is the multi-cells recognition of singular cell which occurs by the multidirectional growing cells or the cells which are long and have various gray level inside. The last miss detection occurs to crowds of cells. This is due to the case of large single cell recognition of multi-cells. It happens because cells are attached and recognized as single cell after the process of binary image. Those main 3 reasons caused error-detections and may affect to cell classification as well. Based on the resultant data of developed standards of hBMSCs and HeLa cells, we could determine cell pattern recognition of hBMSCs in co-culture image with hBMSCs and HeLa cells.

In the development of computerized cell image processing analysis, there are several important steps that need to be implemented, such as preparing suitable specimens, loading, positioning, and focusing the specimens in the microscope, digitizing an image with suitable resolution and spectral properties, finding areas of interest for the analysis, 
segmenting the images, extracting useful features, and finally recognizing the diagnostic or other information of interest through classification or pattern recognition techniques on the cellular or specimen level.

In this paper, we concentrate on the crucial image-pattern classification step. However, the cells are a lot more challenging such as cell growth and differentiation. Thus, fully automatic cell image analysis method are needed. Other researchers have recently developed and suggested the method for quantifying cell formation associated with green fluorescent protein (GFP)-Rac1 translocation in individual cells. This image of the cell nuclei was first segmented, and the result was used as seeds to simplify the segmentation of the cytoplasms (Lockett et al., 1998)

Combining shape and intensity with the use of morphological filtering for good solution has some problems when segmenting bright-field microscopic images of cells. In order to track cultured cells as they differentiate to neurons and glial cells, we need a fast and robust segmentation algorithm. There is a distinction between microscopic images and bright-field images. Due to the presence of a visible background, processing of brightfield images is more complex than that of other images. Generally, segmentation is the process of separating the relevant objects in an image from the background. Thus, segmentation is a crucial step since the success of pattern recognition techniques in image processing system is known to be very dependent upon the effectiveness of the preceding segmentation. Likewise, segmentation of living cell images presents a number of challenges. The images must be taken in a non-invasive manner so that the cells may continue to grow (Duda et al., 2001; Wu et al., 1995).

\section{Conclusions}

In this paper, we propose a method to identify growth of hBMSCs and HeLa cells. We compare normal cells with abnormal cells by a series of image processing steps. The results presented that microscopic images through the brightness correction were performed clearer than those without brightness compensation. And the classification of mixed cells was well performed as well, which is expected to be completed with pattern recognition later. Further research will focus on the feature selection and pattern recognition components along with training and testing on a wider range of hBMSCs culture images.

\section{Conflict of Interest}

No potential conflict of interest relevant to this article was reported.

\section{Acknowledgements}

This study was carried out with the financial support of the Agricultural Research and Development Promotion Center (312031-3), Republic of Korea.

\section{References}

Althoff, K., J. Degerman and T. Gustavsson. 2005. Combined segmentation and tracking of neural stem cells. In Image Analysis. pp. 282-291.

Amini, S., D. Veilleux and I. Villemure. 2010. Tissue and cellular morphological changes in growth plate explants under compression. Journal of Biomechanics 43(13): 2582-2588.

Brun, F., A. Accardo, M. Marchini, F. Ortolani, G. Turco and S. Paoletti. 2011. Texture analysis of TEM micrographs of alginate gels for cell microencapsulation. Microscopy Research and Technique 74(1):58-66.

Chan, S. W. K., K. S. Leung and W. S. F. Wong. 1996. An expert system for the detection of cervical cancer cells using knowledge-based image analyzer. Artificial Intelligence in Medicine 8(1):67-90.

Chaudry, Q., S. H. Raza, A. N. Young and M. D. Wang. 2009. Automated renal cell carcinoma subtype classification using morphological, textural and wavelets based features. Journal of Signal Processing Systems for Signal Image and Video Technology 55:15-23.

Cheng, J. Z., Y. H. Chou, C. S. Huang, Y. C. Chang, C. M. Tiu, F. C. Yeh, K. W. Chen, C. H. Tsou and C. M. Chen. 2010. ACCOMP: Augmented cell competition algorithm for breast lesion demarcation in sonography. Medical Physics 37(12):6240-6252.

Diaz, E., G. Ayala, M. E. Diaz, L. W. Gong and D. Toomre. 2010. Automatic detection of large dense-core vesicles in secretory cells and statistical analysis of their intracellular distribution. IEEE-Acm Transactions on Computational Biology and Bioinformatics 7(1):2-11.

Duda, R. O., P. E. Hart and D. G. Stock. 2001. Pattern Classification, $2^{\text {nd }}$, New York: Wiley-Interscience.

Garrido, A. and N. PeHrez de la Blanca. 2000. Applying 
deformable templates for cell image segmentation. Pattern Recognition 33(5):821-832.

Huang, P. W. and Y. H. Lai. 2010. Effective segmentation and classification for HCC biopsy images. Pattern Recognition 43(4):1550-1563.

Kachouie, N. N., L. J. Lee and P. Fieguth. 2005. A probabilistic living cell segmentation model. In ICIP. pp. 137-140.

Kachouie, N. N., P. Fieguth, J. Ramunas and E. Jervis. 2006. Probabilisticmodel-based cell tracking. Int. Journal of Biomedical Imaging. pp. 1-10.

Kachouie, N. N., P. Fieguth and E. Jervis. 2007. Stem-cell localization: A deconvolution problem. In EMBS. pp. 5525-5528.

Korzynska, A.. 2007. Automatic counting of neural stem cells growing in cultures. In Computer Recognition Systems. pp. 604-612.

Li, F. H., X. B. Zhou, J. W. Ma and S. T. C. Wong. 2010. Multiple nuclei tracking using integer programming for quantitative cancer cell cycle analysis. IEEE Transactions on Medical Imaging 29(1):96-105.

Lockett, S. J., D. Sudar and C. T. Thompson.1998. Efficient, interactive, and three-dimensional Segmentation of Cell Nuclei in Thick Tissue Sections. Cytometry 31: 275-286.

Long, X., W. L. Cleveland and Y. L. Yao. 2005. Effective automatic recognition of cultured cells in bright field images using fisher's linear discriminant preprocessing. Image and Vision Computing 23(13):1203-1213.

Markiewicz, T., S. Osowski, J. Patera and W. Kozlowski. 2006. Image processing for accurate cell recognition and count on histologic slides. Int. Academy of Cytology and American Society of Cytology 28(5):281-291.

Reyes-Aldasoro, C. C., L. J. Williams, S. Akerman, C. Kanthou and G. M. Tozer. 2011. An automatic algorithm for the segmentation and morphological analysis of microvessels in immunostained histological tumour sections. Journal of Microscopy 242(3):262-278.

Plissiti, M. E., C. Nikou and A. Charchanti. 2011. Combining shape, texture and intensity features for cell nuclei extraction in Pap smear images. Pattern Recognition Letters 32(6):838-853.

Schildkraut, J. S., N. Prosser, A. Savakis, J. Gomez, D. Nazareth, A. K. Singh and H. K. Malhotra. 2010. Levelset segmentation of pulmonary nodules in megavolt electronic portal images using a CT prior. Medical Physics 37(11):5703-5710.

Shiotani, S., T. Fukuda, F. Arai, N. Takeuchi, K. Sasaki and T. Kinoshita. 1994. Cell recognition by image processing: (recognition of dead or living plant cells by neural network). JSME 37:202-208.

Spencer, T., J. A. Olson, K. C. Mchardy, P. R. Sharp and J. V. Forrester. 1996. An image-processing strategy for the segmentation and quantification of microaneurysms in fluorescein angiograms of the ocular fundus. Computers and Biomedical Researches 29:284-302.

Tang, C. and E. Bengtsson. 2005. Segmentation and tracking of neural stem cell. In Advances in Intelligent Computing. pp. 851-859.

Wu, K., D. Gauthier and M. D. Levine. 1995. Live cell image segmentation. IEEE Transactions on Biomedical Engineering 42:1-12.

Xiong, Y., C. Kabacoff, J. Franca-Koh, P. N. Devreotes, D. N. Robinson and P. A. Iglesias. 2010. Automated characterization of cell shape changes during amoeboid motility by skeletonization. Bmc Systems Biology, vol. 4 .

Zheng, Q., B. K. Milthorpe and A. S. Jones. 2004. Direct neural network application for automated cell recognition. Cytometry A 57(1):1-9. 